\title{
The function and mechanisms of action of LOXL2 in cancer (Review)
}

\author{
LINGHONG WU and YING ZHU \\ Department of Infectious Diseases, The First Affiliated Hospital of Dalian Medical University, \\ Dalian, Liaoning 116011, P.R. China
}

Received May 31, 2015; Accepted August 26, 2015

DOI: 10.3892/ijmm.2015.2337

\begin{abstract}
The lysyl oxidase (LOX) family is comprised of five members, and some members have recently emerged as important regulators of tumor progression. Among these, at present, LOX-like (LOXL)2 is the prototypical LOX and the most comprehensively studied member. A growing body of evidence has implicated LOXL2 in the promotion of cancer cell invasion, metastasis and angiogenesis, as well as in the malignant transformation of solid tumors. Moreover, a high expression of LOXL2 is associated with a poor prognosis. These data have piqued the interest of a number of researchers and research groups, who have identified LOXL2 as a strong target candidate in the development of inhibitors for use as functional and efficacious tumor therapeutics. In the present study, we summarize the recent progress made regarding LOXL2, mainly focusing on its function and mechanisms of action in tumor progression and metastasis. In this review, we note that LOXL2 promotes tumor progression possibly by activating multiple signal pathways through a variety of mechanisms, both biochemical and biomechanical. The data presented herein may open new avenues for the therapeutic utility of LOXL2.
\end{abstract}

\section{Contents}

1. Introduction

2. Functions of LOXL2 in cancer

3. Mechanisms of action of LOXL2 in cancer

4. Future perspectives

\section{Introduction}

LOX-like (LOXL)2 is a member of the lysyl oxidase (LOX) family, which is comprised of five members, the prototypical LOX and four related members, LOXL1-4. It is a secreted

Correspondence to: Dr Ying Zhu, Department of Infectious Diseases, The First Affiliated Hospital of Dalian Medical University, 222 Zhongshan Road, Xigang, Dalian, Liaoning 116011, P.R. China E-mail: zhuyingsh52@126.com

Key words: lysyl oxidase-like 2, function, mechanism, invasion, metastasis, tumor copper-dependent amine oxidase, and its main role is to catalyze the covalent cross-link of collagen and elastin in the extracellular matrix (ECM). This occurs through the oxidative deamination of peptidyl lysine residues in ECM components $(1,2)$. Each member of the LOX family has a highly conserved carboxyl (C)-terminal domain that contains a copper-binding motif, lysine tyrosylquinone (LTQ) residues and a cytokine receptor-like (CRL) domain, which is essential for catalytic activity (3). The amino-terminal regions are different and are thought to be important in protein-protein interactions. The prodomains in LOX and LOXL1 enable their secretion as inactive proenzymes, which are then activated extracellularly. However, LOXL2, LOXL3 and LOXL4 contain four scavenger receptor cysteine-rich (SRCR) domains instead of a pro-sequence, and SRCR domains are thought to be involved in protein-protein interactions at the $\mathrm{N}$-terminal region (4), and in the ligand binding of both soluble proteins and membrane-bound protein receptors $(5,6)$. Due to similarities in the domain arrangement, LOX and LOXL1 represent one LOX subfamily, whereas LOXL2-4 constitute another LOX subfamily (Fig. 1).

In addition, the presence of various protein domains in LOX family members suggests their involvement in multiple biological functions beyond ECM stabilization. Although all members are involved in different stages of tumor progression (7), and have a high sequence homology, the precise molecular functions of LOXL1, LOXL3, LOXL4 are not yet fully understood. Previous studies on LOXL2 in tumors have, however, made some progress: LOXL2 is currently thought to be involved in developmental regulation, cell adhesion, senescence, cell migration and invasion, metastasis, epithelial-mesenchymal transition (EMT) and malignant transformation $(4,7,8)$. In this review, we summarize the broader areas of the progress made in relation to LOXL2, particularly its functions and mechanisms of action in tumors.

\section{Functions of LOXL2 in cancer}

LOXL2 may be a tumor suppressor. The human LOXL2 gene is located on chromosome 8p21-22, and is initially synthesized as an $87-\mathrm{kDa}$ proenzyme of 774 amino acids. In 1997, the LOXL2 gene was originally identified as a downregulated transcript in various non-adherent tumor cell lines when compared to adherent tumor cell lines (9). Subsequently, several studies have also identified LOXL2 as a downregulated transcript in cell lines and tumors. Investigations into the function of LOXL2 have indicated that the gene is a candidate for tumor suppression (7). 


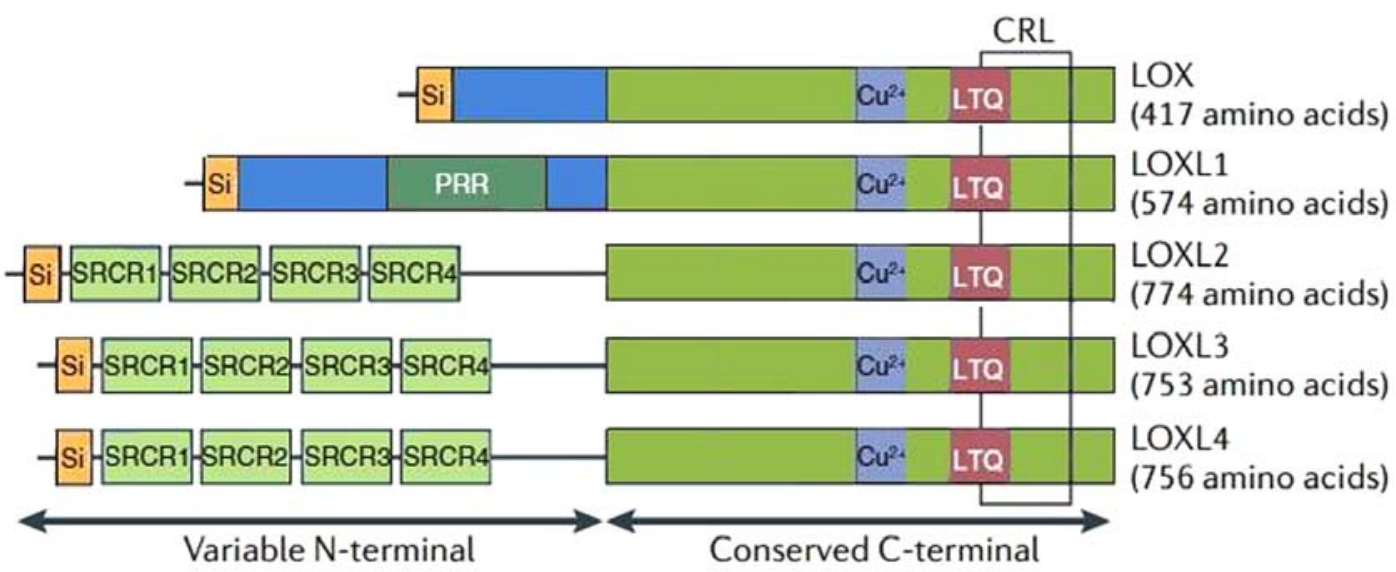

Figure 1. Structure of lysyl oxidase (LOX) family members. All members are highly conserved at their C- terminal mature domains, including the copper binding site $(\mathrm{Cu}+)$, the lysine tyrosylquinone (LTQ) cofactor residues, and the cytokine receptor-like (CRL) domain, but their N-terminal pro-peptides are variable and may play a role in substrate specificity and function. LOX and LOXL (lysyl oxidase-like)1 contain pro-sequences, especially the proline-rich region in LOXL1. LOXL2-4 contain four scavenger receptor cysteine-rich (SRCR) domains that are thought to be involved in protein-protein interactions. Si, signal peptide.

There are several reports that support a role for LOXL2 as a tumor suppressor. A nearly 60-fold decrease in LOXL2 mRNA expression was found to have occurred in RAS-transformed rat fibroblasts (10). The study by Rost et al (11) demonstrated that the downregulation of LOXL2 mRNA expression was observed in several cell lines and tissue samples of head and neck squamous cell carcinoma (HNSCC). Via a large-scale serial analysis of gene expression, downregulated LOXL2 expression was also observed in ovarian tumors $(12,13)$. Recently, a study on non-small cell lung cancer (NSCLC) by Zhan et al (14) revealed that, compared with the surrounding normal tissues, LOXL2 mRNA and protein expression levels were downregulated in tumor samples from patients with NSCLC. However, the upregulation LOXL2 of expression has been reported in breast, colon, esophageal and pancreatic carcinoma cell lines, and gastric cancer $(15,16-18)$. Despite being classed as a secreted protein, LOXL2 has been shown to have both intracellular and extracellular functions, and can induce EMT. These seemingly opposing reports suggest that LOXL2 has complex and paradoxical roles and may function both as a tumor suppressor and promoter of metastasis, depending on its localization and tumor type, and also has the ability to induce EMT (19).

LOXL2 promotes invasion and metastasis in cancer. In contrast to the above-mentioned findings, it has been reported by several groups that the mRNA and protein levels of LOXL2 are increased in invasive or metastatic breast, pancreatic and liver carcinoma cell lines $(17,20,21)$. LOXL2 has been characterized as a promoter of tumor cell invasiveness (22). Moreover, in immunocompetent or immunocompromised orthotopic and transgenic breast cancer models, the genetic, chemical or antibody-mediated inhibition of LOXL2 has been shown to result in decreased metastasis (15). These studies and the emerging evidence suggests that in these situations, LOXL2 may function as a promoter of invasion and metastasis rather than a tumor suppressor.

In 2009, Brekhman and Neufeld (23), using a novel asymmetric 3D in-vitro invasion assay and a quantitative analysis of the directional invasive behavior of tumor cells in a 3D envi-

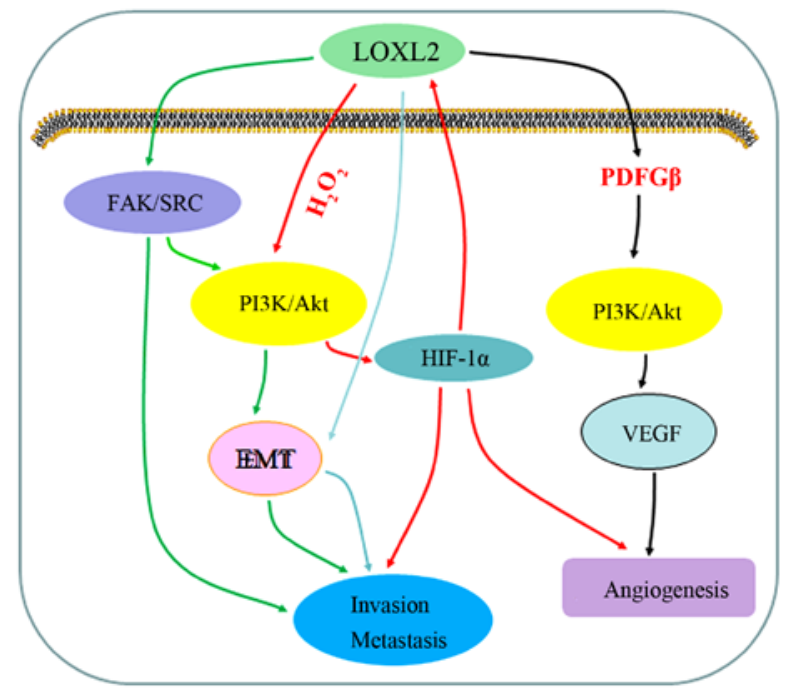

Figure 2. Lysyl oxidase-like (LOXL)2 regulates multiple signaling networks in cancer. Its action is associated with invasion, metastasis and angiogenesis in tumors. FAK, focal adhesion kinase; PI3K/AKT, phosphatidylinositol 3-kinase/protein kinase B; HIF1 $\alpha$, hypoxia-inducible factor $1 \alpha$; VEGF, vascular endothelial growth factor; EMT, epithelial-mesenchymal transition.

ronment mimicking the tumor microenvironment, found that the inhibition of endogenous LOXL2 expression in several types of tumor cells strongly inhibited their invasiveness, and further demonstrated that LOXL2 was a potent inducer of invasiveness. In 2013, a study by Ahn et al (20), using an immunohistochemical study of tissue specimens from 309 patients with breast cancer and reverse transcription polymerase chain reaction (RT-PCR) analysis of human breast cancer cell lines, confirmed that a higher LOXL2 expression was associated with the invasiveness of basal-like breast cancer cells and lower survival rates of breast cancer patients. Subsequently, in 2014, Wong et al (21), integrating the clinical relevance, molecular regulation and functional implications, demonstrated that LOXL2 promoted intrahepatic metastasis and extrahepatic metastasis. The relevant mechanisms are discussed below and are summarized in Fig. 2. 
LOXL2 promotes cancer-associated fibrosis. Fibrosis may play a role in tumor progression by promoting cell transformation and metastasis directly (24). Several studies on the extracellular functions of the LOX family in physiological and pathological processes have found that the LOX family plays a critical role in organs containing fibrillar collagen and/or elastic fibers, such as the skin, lungs, the cardiovascular system, cartilage, and the fibrous lamina propria in the small intestines and stomach (25-27). For example, when LOXL1 is knocked down, normal elastic fibers are not deposited in the uterine tract postpartum, and mice lacking LOXL1 develop pelvic organ prolapse, enlarged air spaces in the lungs, loose skin and vascular abnormalities with concomitant tropoelastin accumulation (27). In clinical practice, a reduced LOX activity owing to the diminished availability of copper, was found in two X-linked recessively inherent disorders, Menkes disease and occipital horn syndrome (28). The above-mentioned findings indicate that LOXL2 promotes fibrosis. Indeed, accumulating copper upregulates the expression of LOXL2 in Wilson's disease, and then leads to liver fibrosis (29). In fibrotic processes, an excessive deposition of collagen in particular, changes the physical, biochemical and biomechanical matrix properties, which eventually leads to defective organ function and organ failure (30). Moreover, targeting LOXL2 with an inhibitory monoclonal antibody (AB0023) has been shown to be efficacious in both primary and metastatic xenograft models of cancer, as well as in models of liver and lung fibrosis (31).

LOXL2 is a prognostic marker for cancer. LOXL2 was, for the first time, described as a prognostic marker for laryngeal squamous cell carcinomas (32). Peinado et al (32) examined the protein expression of LOXL2 by immunohistochemistry in a cohort of 256 human laryngeal squamous cell carcinomas, and found that increased expression of LOXL2 correlated with local recurrence, and moreover, that high levels of LOXL2 were associated with decreased overall and disease-free survival in patients with laryngeal squamous cell carcinomas, lung squamous cell carcinoma and lymph node-negative (N0) breast adenocarcinomas. Subsequently, several studies demonstrated that the high expression or overexpression of LOXL2 correlated with a poor prognosis and decreased survival in gastric cancer (18), colon tumor (16,33), basal-like breast carcinoma (34), pancreatic carcinoma (17) and hepatocellular carcinoma (21).

\section{Mechanisms of action of LOXL2 in cancer}

LOXL2 promotes the activation offocal adhesion kinase (FAK)/ Src in cancer. The involvement of LOXL2 in tumor cell invasion and metastasis provides a powerful intervention tool for the prevention of tumor cell dissemination. A previous study on the underlying mechanisms through which LOXL2 contributes to tumor progression demonstrated that LOXL2 expression promotes the activation of associated Src kinase and FAK, and leads to the activation of associated downstream signaling cascades (18). Indeed, studies have suggested that Src and FAK are activated via LOX-mediated increased collagen crosslinking, resulting in increased matrix stiffness, which is affected by transmembrane integrins that then stimulate the downstream activation of FAK and Src $(35,36)$. In addition, the activation of Src and FAK increases tumor cell proliferation and invasion and is related to the formation of distant metastasis $(18,35,36)$. Moreover, using anti-LOXL2 antibody has been shown to suppress the activity of FAK and Src, and to prevent gastric cancer metastases, thus confirming the mechanisms of action of LOXL2 (18).

LOXL2 increases the expression of vascular endothelial growth factor (VEGF) in cancer. Angiogenesis contributes to primary and metastatic cancer growth, and it is necessary for tumor progression $(37,38)$. The VEGF signaling pathway plays a key role in promoting angiogenesis, not only under normal conditions, but also in tumor pathogenesis. Thus, it has become a major intervention tool in targeted cancer therapy $(39,40)$. A study on the effects of LOX expression on tumor-driven angiogenesis, demonstrated that the regulation of VEGF mRNA and protein expression is carried out through the platelet-derived growth factor $\beta$ (PDGFR $\beta$ )-mediated activation of protein kinase $\mathrm{B}$ (Akt) (41). In clinical practice, the use of angiogenesis inhibitors (bevacizumab and sunitinib) or PDGF $\beta$ - or Akt-mediated signaling inhibitors, has been shwon to prevent the LOX-mediated increase in VEGF mRNA and protein expression, and to abrogate the LOX-mediated stimulation of endothelial cells both in vivo and in vitro (41). Moreover, a recent report suggested that the inhibition of LOXL2 activity inhibits angiogenesis in part by affecting VEGF signaling in endothelial cells (42).

LOXL2 and hypoxia-inducible factor-1 $\alpha(H I F-1 \alpha)$ reinforce each other in cancer. Several human cancers contain hypoxic regions. Tumor cells adapt to hypoxic environments better than normal cells (43). Under hypoxic conditions, the hypoxia transcription factor, HIF-1 $\alpha$, binds to the hypoxia responsive element (HRE) in the promoter region of numerous target genes, including LOX and LOXL2 $(44,45)$. A previous study using chromatin immunoprecipitation (ChIP) with rabbit polyclonal antiserum to HIF-1 $\alpha$ suggested that LOXL2 is also significantly affected by HIF-1 $\alpha$ (46). LOX overexpression increases HIF-1 $\alpha$ expression under hypoxic conditions; the LOX-induced increase in HIF-1 $\alpha$ expression means they reinforce each other to promote tumor progression (47); tumor cells have greater proliferative, invasive and metastatic abilities and express higher levels of LOX or LOXL2 $(23,46)$. The tumor hypoxic environment can also drive LOXL2 to be involved in angiogenesis. During angiogenesis, LOXL2 contributes to the reorganization of collagen and fibronectin associated with ECM remodeling by HIF-1 $\alpha$ (48).

LOXL2 is associated withphosphatidylinositol3-kinase (PI3K)/ Akt in cancer. A previous study (47) suggested a mechanism of LOX, namely that it regulates $\mathrm{H}_{2} \mathrm{O}_{2}$ production, a by-product of the enzymatic reaction, and activates the signaling pathway of PI3K/Akt, then increases HIF-1 $\alpha$ protein synthesis; thus LOX production further constitutes a positive regulation loop and promotes tumor proliferation. In a breast cancer model, LOX-mediated $\mathrm{H}_{2} \mathrm{O}_{2}$ production has also been shown to participate in the activation of Src and FAK (49). Indeed, FAK and Src are upstream signaling cascade components of PI3K (50). LOXL2 can also produce $\mathrm{H}_{2} \mathrm{O}_{2}$ on the regeneration of catalytic activity and the presence of $\mathrm{H}_{2} \mathrm{O}_{2}$ can promote Src and FAK activation (18). 
LOXL2 drives EMT in cancer. EMT, an essential process in development, is described as a key event in tumor progression, facilitating the dissociation of cancer cells from the primary tumors concomitant to the acquisition of cell migration that provides tumor cells with the ability to invade into the adjacent tissues (51). Moreover, EMT is considered to be relevant for other stages of the metastatic cascade, such as intravasation or extravasation (52). EMT is currently considered as a focal and transient event occurring at specific tumor regions and being perhaps more relevant to specific tumor types (51-53). Indeed, LOXL2 is originally described as affirmatively promoting EMT inducer Snail1 stability and functional activity, driving EMT and tumor progression through the downregulation of E-cadherin expression at the transcriptional level (54). A previous study reported that LOXL2 also mediates EMT through a Snail1-independent mechanism, related to the downregulation of epidermal differentiation and cell polarity components (34). Recently, Cuevas et al provided evidence that catalytically inactive LOXL2 mutants, one of them unable to be secreted, induce and sustain a full EMT process, indicating that intracellular LOXL2 drives EMT independent of its enzymatic activity (55). All the above-mentioned data support the hypothesis that LOXL2 induces EMT.

Of note, previous studies have demonstrated that some factors trigger EMT by activating the PI3K/Akt signaling pathway (56-58), and thus, LOXL2 can also possibly induce EMT through PI3K/Akt. Thereby, LOXL2 promotes tumor progression possibly by activating multiple signal pathways through a variety of mechanisms, both biochemical and biomechanical. Thus, we propose that the mechanisms of action of LOXL2 are applicable to many common cancer models (Fig. 2), meaning that solid tumors have a high commonality.

\section{Future perspectives}

A malignant tumor is frequently diagnosed at an advanced stage, as a result of tumor cell invasion and metastases, and is usually indicative of a poor prognosis for patients. The diagnosis of advanced stage of carcinoma is a devastating experience for both patients and their families. The important role of LOXL2 in a number of common tumors is unquestionable. Its effects on cell invasion, metastasis, and the induction of angiogenesis and malignant transformation have increased its viability as a possible target for therapeutic intervention. For instance, a study group from California demonstrated that a monoclonal antibod (AB0023) against LOXL2 was efficacious in both primary and metastatic xenograft models (31). Moreover, AB0024, which is a novel humanized LOXL2 antibody, has already entered phase I clinical trials in patients with solid tumors (ClinicalTrials.gov.) (59). Even though some molecular-targeted drugs have been developed, their effects have not yet reached our expectations. Therefore, more comprehensive studies are warranted to broaden our understanding of the mechanisms through which LOXL2 modulates the cancer microenvironment and exerts its promoting effects on cancer progression and metastasis, and to develop novel anticancer therapeutics. Although the future is full of challenges, the development of inhibitors targeting LOXL2 brings us hope that effective treatments for primary and metastatic tumors will be feasible in the near future.

\section{References}

1. Kagan HM and Trackman PC: Properties and function of lysyl oxidase. Am J Respir Cell Mol Biol 5: 206-210, 1991.

2. Smith-Mungo LI and Kagan HM: Lysyl oxidase: properties, regulation and multiple functions in biology. Matrix Biol 16: 387-398, 1998.

3. Lopez KM and Greenaway FT: Identification of the copper-binding ligands of lysyl oxidase. J Neural Transm 118: 1101-1109, 2011.

4. Lucero HA and Kagan HM: Lysyl oxidase: an oxidative enzyme and effector of cell function. Cell Mol Life Sci 63: 2304-2316, 2006.

5. Hohenester E, Sasaki T and Timpl R: Crystal structure of a scavenger receptor cysteine-rich domain sheds light on an ancient superfamily. Nat Struct Biol 6: 228-232, 1999.

6. Martínez VG, Moestrup SK, Holmskov U, Mollenhauer J and Lozano F: The conserved scavenger receptor cysteine-rich superfamily in therapy and diagnosis. Pharmacol Rev 63: 967-1000, 2011.

7. Payne SL, Hendrix MJ and Kirschmann DA: Paradoxical roles for lysyl oxidases in cancer - a prospect. J Cell Biochem 101: 1338-1354, 2007.

8. Barker HE, Cox TR and Erler JT: The rationale for targeting the LOX family in cancer. Nat Rev Cancer 12: 540-552, 2012.

9. Saito H, Papaconstantinou J, Sato H and Goldstein S: Regulation of a novel gene encoding a lysyl oxidase-related protein in cellular adhesion and senescence. J Biol Chem 272: 8157-8160, 1997.

10. Zuber J, Tchernitsa OI, Hinzmann B, Schmitz AC, Grips M, Hellriegel M, Sers C, Rosenthal A and Schäfer R: A genome-wide survey of RAS transformation targets. Nat Genet 24: 144-152, 2000.

11. Rost T, Pyritz V, Rathcke IO, Görögh T, Dünne AA and Werner JA: Reduction of LOX- and LOXL2-mRNA expression in head and neck squamous cell carcinomas. Anticancer Res 23 (2B): 1565-1573, 2003.

12. Hough CD, Sherman-Baust CA, Pizer ES, Montz FJ, Im DD, Rosenshein NB, Cho KR, Riggins GJ and Morin PJ: Large-scale serial analysis of gene expression reveals genes differentially expressed in ovarian cancer. Cancer Res 60: 6281-6287, 2000.

13. Ono K, Tanaka T, Tsunoda T, Kitahara O, Kihara C, Okamoto A, Ochiai K, Takagi $\mathrm{T}$ and Nakamura Y: Identification by cDNA microarray of genes involved in ovarian carcinogenesis. Cancer Res 60: 5007-5011, 2000

14. Zhan P, Shen XK, Qian Q, Zhu JP, Zhang Y, Xie HY, Xu CH, Hao KK, Hu W, Xia N, et al: Down-regulation of lysyl oxidase-like 2 (LOXL2) is associated with disease progression in lung adenocarcinomas. Med Oncol 29: 648-655, 2012.

15. Barker HE, Chang J, Cox TR, Lang G, Bird D, Nicolau M, Evans HR, Gartland A and Erler JT: LOXL2-mediated matrix remodeling in metastasis and mammary gland involution. Cancer Res 71: 1561-1572, 2011.

16. Fong SF, Dietzsch E, Fong KS, Hollosi P, Asuncion L, He Q, Parker MI and Csiszar K: Lysyl oxidase-like 2 expression is increased in colon and esophageal tumors and associated with less differentiated colon tumors. Genes Chromosomes Cancer 46: 644-655, 2007.

17. Rückert F, Joensson P, Saeger HD, Grützmann R and Pilarsky C: Functional analysis of LOXL2 in pancreatic carcinoma. Int $\mathrm{J}$ Colorectal Dis 25: 303-311, 2010.

18. Peng L, Ran YL, Hu H, Yu L, Liu Q, Zhou Z, Sun YM, Sun LC, Pan J, Sun LX, et al: Secreted LOXL2 is a novel therapeutic target that promotes gastric cancer metastasis via the Src/FAK pathway. Carcinogenesis 30: 1660-1669, 2009.

19. Cano A, Santamaría PG and Moreno-Bueno G: LOXL2 in epithelial cell plasticity and tumor progression. Future Oncol 8: 1095-1108, 2012.

20. Ahn SG, Dong SM, Oshima A, Kim WH, Lee HM, Lee SA, Kwon SH, Lee JH, Lee JM, Jeong J, et al: LOXL2 expression is associated with invasiveness and negatively influences survival in breast cancer patients. Breast Cancer Res Treat 141: 89-99, 2013.

21. Wong CC, Tse AP, Huang YP, Zhu YT, Chiu DK, Lai RK, Au SL, Kai AK, Lee JM, Wei LL, et al: Lysyl oxidase-like 2 is critical to tumor microenvironment and metastatic niche formation in hepatocellular carcinoma. Hepatology 60: 1645-1658, 2014.

22. Akiri G, Sabo E, Dafni H, Vadasz Z, Kartvelishvily Y, Gan N, Kessler O, Cohen T, Resnick M, Neeman M and Neufeld G: Lysyl oxidase-related protein-1 promotes tumor fibrosis and tumor progression in vivo. Cancer Res 63: 1657-1666, 2003. 
23. Brekhman V and Neufeld G: A novel asymmetric 3D in-vitro assay for the study of tumor cell invasion. BMC Cancer 9: 415, 2009.

24. Cox TR and Erler JT: Remodeling and homeostasis of the extracellular matrix: Implications for fibrotic diseases and cancer. Dis Model Mech 4: 165-178, 2011.

25. Mäki JM, Räsänen J, Tikkanen H, Sormunen R, Makikallio K, Kivirikko KI and Soininen R: Inactivation of the lysyl oxidase gene Lox leads to aortic aneurysms, cardiovascular dysfunction, and perinatal death in mice. Circulation 106: 2503-2509, 2002.

26. Mäki JM, Sormunen R, Lippo S, Kaarteenaho-Wiik R, Soininen R and Myllyharju J: Lysyl oxidase is essential for normal development and function of the respiratory system and for the integrity of elastic and collagen fibers in various tissues.Am J Pathol 167: 927-936, 2005

27. Liu X, Zhao Y, Gao J, Pawlyk B, Starcher B, Spencer JA, Yanagisawa $\mathrm{H}$, Zuo $\mathrm{J}$ and $\mathrm{Li} \mathrm{T}$ : Elastic fiber homeostasis requires lysyl oxidase-like 1 protein. Nat Genet 36: 178-182, 2004.

28. Mäki JM: Lysyl oxidases in mammalian development and certain pathological conditions. Histol Histopathol 24: 651-660, 2009

29. Vadasz Z, Kessler O, Akiri G, Gengrinovitch S, Kagan HM, Baruch Y, Izhak OB and Neufeld G: Abnormal deposition of collagen around hepatocytes in Wilson's disease is associated with hepatocyte specific expression of lysyl oxidase and lysyl oxidase like protein-2. J Hepatol 43: 499-507, 2005.

30. Nieto MA: The ins and outs of the epithelial to mesenchymal transition in health and disease. Annu Rev Cell Dev Biol 27: 347-376, 2011.

31. Barry-Hamilton V, Spangler R, Marshall D, McCauley S, Rodriguez HM, Oyasu M, Mikels A, Vaysberg M, Ghermazien H, Wai C, et al: Allosteric inhibition of lysyl oxidase-like-2 impedes the development of a pathologic microenvironment. Nat Med 16: 1009-1017, 2010.

32. Peinado H, Moreno-Bueno G, Hardisson D, Pérez-Gómez E, Santos V, Mendiola M, de Diego JI, Nistal M, Quintanilla M, Portillo F and Cano A: Lysyl oxidase-like 2 as a new poor prognosis marker of squamous cell carcinomas. Cancer Res 68: 4541-4550, 2008.

33. Offenberg H, Brünner N, Mansilla F, Orntoft Torben F and Birkenkamp-Demtroder K: TIMP-1 expression in human colorectal cancer is associated with TGF-B1, LOXL2, INHBA1, TNF-AIP6 and TIMP-2 transcript profiles. Mol Oncol 2: 233-240, 2008

34. Moreno-Bueno G, Salvador F, Martín A, Floristán A, Cuevas EP, Santos V, Montes A, Morales S, Castilla MA, Rojo-Sebastián A, et al: Lysyl oxidase-like 2 (LOXL2), a new regulator of cell polarity required for metastatic dissemination of basal-like breast carcinomas. EMBO Mol Med 3: 528-544, 2011

35. Baker AM, Cox TR, Bird D, Lang G, Murray GI, Sun XF, Southall SM, Wilson JR and Erler JT: The role of lysyl oxidase in SRC-dependent proliferation and metastasis of colorectal cancer. J Natl Cancer Inst 103: 407-424, 2011.

36. Baker AM, Bird D, Lang G, Cox TR and Erler JT: Lysyl oxidase enzymatic function increases stiffness to drive colorectal cancer progression through FAK. Oncogene 32: 1863-1868, 2013.

37. Ferrara $\mathrm{N}$ and Kerbel RS: Angiogenesis as a therapeutic target. Nature 438: 967-974.

38. Kerbel RS: Tumor angiogenesis. N Engl J Med 358: 2039-2049, 2008

39. Jubb AM and Harris AL: Biomarkers to predict the clinical efficacy of bevacizumab in cancer. Lancet Oncol 11: 1172-1183, 2010.

40. Kerbel R and Folkman J: Clinical translation of angiogenesis inhibitors. Nat Rev Cancer 2: 727-739, 2002.

41. Baker AM, Bird D, Welti JC, Gourlaouen M, Lang G, Murray GI, Reynolds AR, Cox TR and Erler JT: Lysyl oxidase plays a critical role in endothelial cell stimulation to drive tumor angiogenesis. Cancer Res 73: 583-594, 2013.

42. Zaffryar-Eilot S, Marshall D, Voloshin T, Bar-Zion A, Spangler R, Kessler O, Ghermazien H, Brekhman V, Suss-Toby E, Adam D, et al: Lysyl oxidase-like-2 promotes tumour angiogenesis and is a potential therapeutic target in angiogenic tumours. Carcinogenesis 34: 2370-2379, 2013.
43. Cassavaugh $\mathrm{J}$ and Lounsbury KM: Hypoxia-mediated biologica control. J Cell Biochem 112: 735-744, 2011

44. Wang GL, Jiang BH, Rue EA and Semenza GL: Hypoxia-inducible factor 1 is a basic-helix-loop-helix-PAS heterodimer regulated by cellular O2 tension. Proc Natl Acad Sci USA 92: 5510-5514, 1995.

45. Postovit LM, Abbott DE, Payne SL, Wheaton WW, Margaryan NV, Sullivan R, Jansen MK, Csiszar K, Hendrix MJ and Kirschmann DA: Hypoxia/reoxygenation: A dynamic regulator of lysyl oxidase-facilitated breast cancer migration. J Cell Biochem 103: 1369-1378, 2008.

46. Schietke R, Warnecke C, Wacker I, Schödel J, Mole DR, Campean V, Amann K, Goppelt-Struebe M, Behrens J, Eckardt KU and Wiesener MS: The lysyl oxidases LOX and LOXL2 are necessary and sufficient to repress E-cadherin in hypoxia: insights into cellular transformation processes mediated by HIF-1. J Biol Chem 285: 6658-6669, 2010.

47. Pez F, Dayan F, Durivault J, Kaniewski B, Aimond G, Le Provost GS, Deux B, Clézardin P, Sommer P, Pouysségur J and Reynaud C: The HIF-1-inducible lysyl oxidase activates HIF-1 via the Akt pathway in a positive regulation loop and synergizes with HIF-1 in promoting tumor cell growth. Cancer Res 71: 1647-1657, 2011.

48. Bignon M, Pichol-Thievend C, Hardouin J, Malbouyres M, Bréchot N, Nasciutti L, Barret A, Teillon J, Guillon E, Etienne E, et al: Lysyl oxidase-like protein-2 regulates sprouting angiogenesis and type IV collagen assembly in the endothelial basement membrane. Blood 118: 3979-3989, 2011.

49. Payne SL, Fogelgren B, Hess AR, Seftor EA, Wiley EL, Fong SF, Csiszar K, Hendrix MJ and Kirschmann DA: Lysyl oxidase regulates breast cancer cell migration and adhesion through a hydrogen peroxide-mediated mechanism. Cancer Res 65: 11429-11436, 2005.

50. Kim BR, Dong SM, Seo SH, Lee JH, Lee JM, Lee SH and Rho SB: Lysyl oxidase-like 2 (LOXL2) controls tumor-associated cell proliferation through the interaction with MARCKSL1. Cell Signal 26: 1765-1773, 2014.

51. Thiery JP, Acloque H, Huang RY and Nieto MA: Epithelial-mesenchymal transitions in development and disease. Cell 139: 871-890, 2009.

52. Polyak K and Weinberg RA: Transitions between epithelial and mesenchymal states: acquisition of malignant and stem cell traits. Nat Rev Cancer 9: 265-273, 2009.

53. Peinado H, Olmeda D and Cano A: Snail, Zeb and bHLH factors in tumour progression: an alliance against the epithelial phenotype? Nat Rev Cancer 7: 415-428, 2007.

54. Peinado H, Del Carmen Iglesias-de la Cruz M, Olmeda D, Csiszar K, Fong KS, Vega S, Nieto MA, Cano A and Portillo F: A molecular role for lysyl oxidase-like 2 enzyme in snail regulation and tumor progression. EMBO J 24: 3446-3458, 2005.

55. Cuevas EP, Moreno-Bueno G, Canesin G, Santos V, Portillo F and Cano A: LOXL2 catalytically inactive mutants mediate epithelial-to-mesenchymal transition. Biol Open 3: 129-137, 2014.

56. Wang F, Ruan XJ and Zhang HY: BDE-99 (2,2',4,4',5-pentabromodiphenyl ether) triggers epithelial-mesenchymal transition in colorectal cancer cells via PI3K/Akt/Snail signaling pathway. Tumori 101: 238-245, 2015.

57. Lin JJ, Zhao TZ, Cai WK, Yang YX, Sun C, Zhang Z, Xu YQ, Chang T and Li ZY: Inhibition of histamine receptor 3 suppresses glioblastoma tumor growth, invasion, and epithelial-to-mesenchymal transition. Oncotarget 6: 17107-17120, 2015.

58. Rafael D, Doktorovová S, Florindo HF, Gener P, Abasolo I, Schwartz S Jr and Videira MA: EMT blockage strategies: Targeting Akt dependent mechanisms for breast cancer metastatic behaviour modulation. Curr Gene Ther 15: 300-312, 2015.

59. ClinicalTrials.gov: First-in-human study of AB0024 to evaluate safety and tolerability in adults with advanced solid tumors. https://clinicaltrials.gov/ct2/show/NCT01323933. Accessed March 25, 2013. 